\title{
Understanding the Slip Planarity and Residual Strain Field in Ti-6Al using Nanobeam Electron Diffraction and First Principles Calculations
}

\author{
Ruopeng Zhang ${ }^{1,2}$, Shiteng Zhao ${ }^{1,2}$, Thomas Pekin ${ }^{3}$, Eric Rothchild ${ }^{1}$, Mark Asta ${ }^{1,4}$, Daryl. C. Chrzan ${ }^{1,4}$ \\ and Andrew M. Minor ${ }^{1,2, *}$ \\ 1. Department of Materials Science and Engineering, University of California, Berkeley, Berkeley \\ 94720, USA \\ 2. National Center for Electron Microscopy, Molecular Foundry, Lawrence Berkeley National \\ Laboratory, Berkeley 94720, USA \\ 3. Department of Physics, Humboldt-Universität zu Berlin, Newtonstraße 15, 12489 Berlin, Germany. \\ 4. Materials Science Division, Lawrence Berkeley National Laboratory, Berkeley 94720, USA \\ * Corresponding author: aminor@berkeley.edu
}

The anomalous solid-solution strengthening and low strain hardening of Ti-Al alloys have long been attributed to the chemical short-range ordering (SRO) of $\mathrm{Al}$ atoms [1-2]. The existence of SRO and its impact on deformation behavior has been revealed through various indirect techniques [3-5]. Zhang et al. directly observed the SRO domain structure with Energy-Filtered Transmission Electron Microscopy (EFTEM), confirming the microstructure and spatial distribution of SRO [6]. As the dominant deformation mode, dislocations slip in Ti-6Al would cut through SRO clusters and, therefore, create "diffuse" antiphase boundaries (DAPB), leading to a localized strain field that has been experimentally observed [6-7]. Despite the successful observation of a reduction in SRO concentration due to dislocation plasticity, the extent and the origin of the residual strain, as well as its relation to the formation of the planar slip, is not straightforward.

With the adoption of nanobeam electron diffraction (NBED), strain mapping with nanometer spatial resolution is possible [8]. Here, we utilized NBED to study the residual strain in slip bands of SRO-aged Ti-6Al. Figure 1 (a) was taken from a dislocation-free section of a slip band, showing the distribution of residual strain of [2 $\overline{1} \overline{1} 0$ ] orientation. One limitation of our previous effort is the lack of 3-D mapping capability. Here, we propose to acquire 3-D strain information by doing a "tilt-series" of strain mapping, followed by reconstructing the 3-D information according to the crystallographic orientations. Previously, the residual strain caused by the formation of three different anti-phase boundaries (APB) in $\mathrm{Ti}_{3} \mathrm{Al}$ structure has been assessed with first principles calculations [9], but a DAPB in a SRO Ti-6Al is much more complicated and requires a more dedicated calculation. Correlating the results of NBED and first principles calculations could provide direct insights into the multiscale origin of slip planarity. The development of fast direct electron detectors has enabled in situ nanomechanical NBED experiments. The formation of planar slip and the strain evolution could be directly observed and analyzed [10].

\section{References:}

[1] M.J. Blackburn and J.C. Williams, Trans. ASM 62 (1969), p. 398-409.

[2] Conrad, H., Scripta Metallurgica 7.5 (1973), p. 509-512.

[3] Namboodhiri, T.K.G., McMahon, C.J. and Herman, H., Metallurgical Transactions, 4(5) (1973), p.1323-1331.

[4] Fitzner, Arnas, et al., Acta Materialia 103 (2016), p. 341-351.

[5] Neeraj, T., and M. J. Mills, Philosophical Magazine A 82.4 (2002), p. 779-802. 
[6] Zhang, R., Traylor, R., Pekin, T., Ozdol, B., Ophus, C., and Minor, A. M. (2018). Microscopy and Microanalysis, 24(S1) (2018), p. 210-211.

[7] Neeraj, T., and M. J. Mills, Philosophical Magazine A 82.4 (2002), p. 779-802.

[8] Pekin, Thomas C., et al., Scripta Materialia 146 (2018), p. 87-90.

[9] Van de Walle, A., and M. Asta, Metallurgical and Materials Transactions A 33.3 (2002), p. 735-741. [10] We gratefully acknowledge funding from the US Office of Naval Research under Grant No.

N00014-12-1-0413. Work at the Molecular Foundry was supported by the Office of Science, Office of Basic Energy Sciences, of the U.S. Department of Energy under Contract No. DE-AC02-05CH11231.

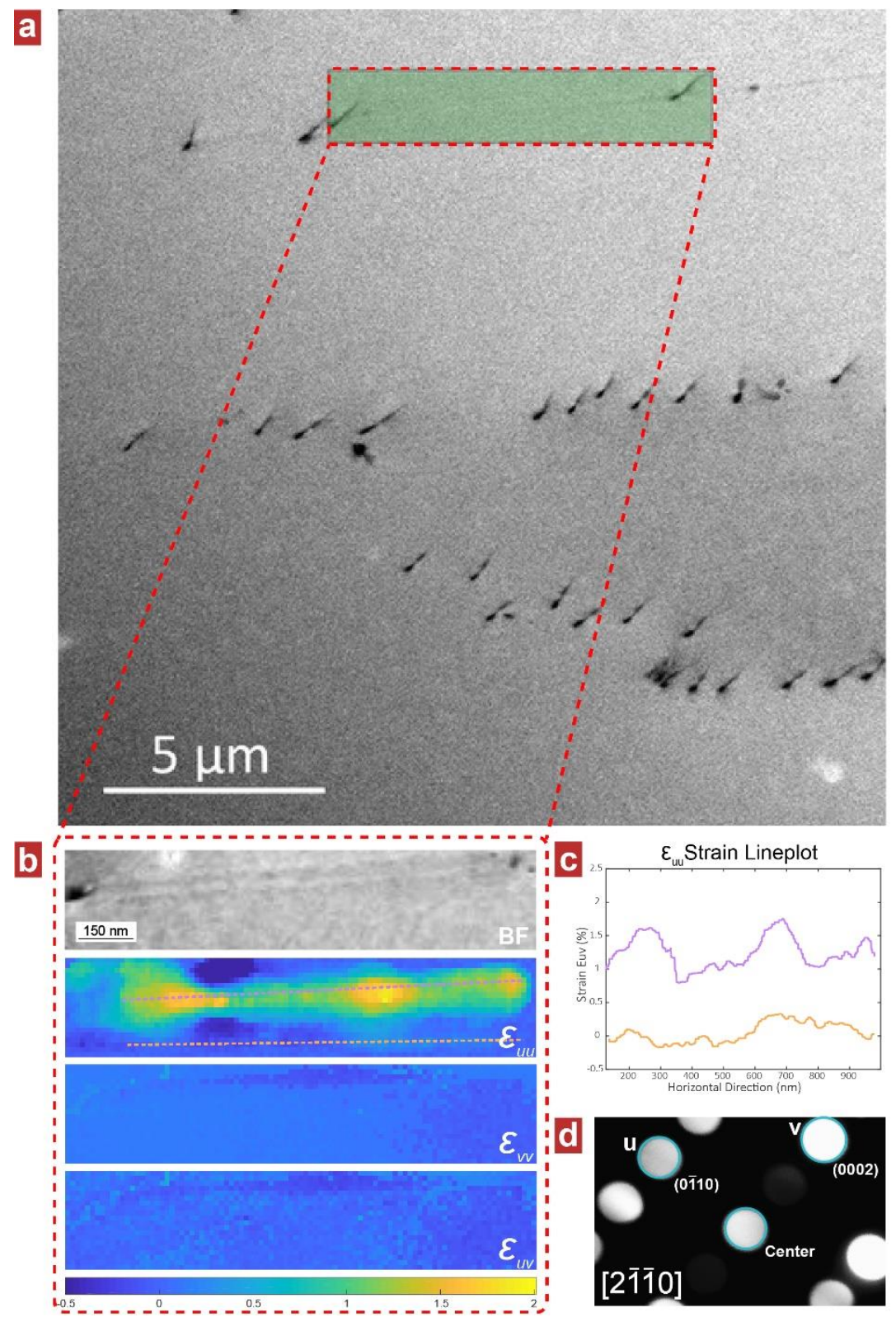

Figure 1. Strain mapping results taken from a dislocation-free slip band; (a), HAADF image showing the region of interest; (b), reconstructed BF image and the 3 different elements of the strain mapping; (c), line plots of strain from in and out of the slip band; (d), averaged diffraction pattern from the nanobeam electron diffraction dataset. 